\title{
Obesity Paradox and Recurrent Coronary Heart Disease in a Population-Based Study: Tehran Lipid and Glucose Study
}

\author{
Samaneh Asgari, ${ }^{1}$ Maryam Barzin, ${ }^{2}$ Farhad Hosseinpanah,,${ }^{*}$ Farzad Hadaegh, ${ }^{1}$ Fereidoun Azizi, ${ }^{3}$ and \\ Davood Khalili ${ }^{1}$ \\ ${ }^{1}$ Prevention of Metabolic Disorders Research Center, Research Institute for Endocrine Sciences, Shahid Beheshti University of Medical Sciences, Tehran, IR Iran \\ ${ }^{2}$ Obesity Research Center, Research Institute for Endocrine Science, Shahid Beheshti University of Medical Sciences, Tehran, IR Iran \\ ${ }^{3}$ Endocrine Research Center, Research Institute for Endocrine Sciences, Shahid Beheshti University of Medical Sciences, Tehran, IR Iran \\ Corresponding author: Farhad Hosseinpanah, Obesity Research Center, Research Institute for Endocrine Sciences, Shahid Beheshti University of Medical Sciences, P. O. Box: \\ 19395476, Tehran, IR Iran. Tel: +98-2122432500; +98-9123431927, Fax: +98-2122416264, E-mail: fhospanah@endocrine.ac.ir
}

Received 2016 February 09; Accepted 2016 March 14.

\begin{abstract}
Background: Although current data shows a positive association between obesity and development of coronary heart disease (CHD) in general population, there is limited data on the important protective role of central or general obesity in patients with prevalent CHD or the "obesity paradox", from this region.

Objectives: The objective of the present investigation was to describe the relationship between BMI categories and the recurrence of CHD in patients with a history of CHD using data from a large population-based study, the Tehran lipid and glucose study (TLGS). Patients and Methods: The study was conducted on 440 adults, aged $\geq 30$ years, with a history of CHD at baseline who attended the first (1999-2001) or second (2001-2003) phases of the Tehran Lipid and Glucose Study and had at least one year of follow-up until March 31, 2010. Cox proportional hazard models were used to evaluate the association among various BMI groups and recurrent CHD incidence.

Results: During a median follow-up of 8.44 years, 169 new cases of CHD occurred (incidence density of: 54.53 per 1000 personyears). The incidence of recurrent CHD was higher in the normal BMI compared with overweight and obese categories (68.71, 47.56 and 54.46 per 1000 person-years, respectively). In multivariable models, using the forward stepwise selection approach, compared to the overweight group (0.48 95\% CI, 0.30-0.80), the obese group (0.55 95\% CI, 0.28-1.06) lost its significant protective effect. Conclusions: Results of this study demonstrated an apparently protective effect for overweight in comparison with normal weight against long-term recurrent CHD in patients with history of CHD.
\end{abstract}

Keywords: Body Mass Index, Obesity, Overweight, Recurrent Coronary Disease, Cohort Study

\section{Background}

Obesity, manifested by body mass index (BMI) cutoffs, is considered a major risk factor not only for type 2 diabetes (1), lipid disorders, and hypertension, but also for coronary heart disease (CHD) (2) and certain cancers (3).

Coronary heart disease continues to be one of the dominant causes of all chronic diseases among adults in America and in most developed countries (4). The incidence of CHD varies from nation to nation, and society to society. Data shows a high prevalence of CHD in the Middle East, with an age adjusted prevalence of CHD about 22\% (5). The crude incidence rate in men is reported to be around 12 per 1000 person-years, approximately twice that in women (6.5 per 1000 person-years)(6).

An epidemiological study from this region found that obesity increased the risk of CHD by about $87 \%$, an ef- fect which remained significant even after metabolic syndrome adjustment (increase the risk about 48\% ) (7). Despite obesity preserve a risk factor for CHD, an inverse relationship between obesity and mortality has been described in patients with heart failure, CHD and diabetes (810).

This unexpected phenomenon, known as the 'obesity survival paradox', assumes that being overweight or even obese provides a mortality advantage, compared to being normal weight (BMI 18.5-24.9 kg/m²).

While data regarding the risks of diabetes and cardiovascular morbidity with increasing BMI are overwhelming, limited attention has been paid to the possibility of an altered BMI nadir or optimal weight for all-cause and cardiovascular mortality in those with established coronary heart disease.

A variety of studies have evaluated obesity paradox in 
overweight and obese patients with CHD and cardiovascular disease (CVD) (11-15 ). Moreover, a systematic review of 40 cohort studies with 250,152 patients found significantly lower risks for cardiovascular mortality (RR 0.88) in overweight patients (5). Some of these opposite reports originate controversial data were from studies that included cohorts of elderly or used data from seriously ill patients.

However, despite the existence of a positive association between obesity and the development of CHD in the general population, the important role of obesity (central or general) in patients with established coronary disease is less defined. A study from the prospective REduction of Atherothrombosis for Continued Health (REACH) registry, demonstrated the protective effect of BMI $\geq 20 \mathrm{~kg} / \mathrm{m}^{2}$ on recurrent CVD (16). To the best of our knowledge there is no study from the Middle East regarding the effect of overweight/obesity regarding recurrent CHD.

\section{Objectives}

Given the existing controversy on obesity, the objective of this investigation was to determine the relationship between BMI categories and recurrence of coronary heart disease in patients with history of CHD using data from a large population based study, the Tehran Lipid and Glucose Study (TLGS).

\section{Patients and Methods}

The Tehran Lipid and Glucose Study is an ongoing population-based cohort, designed to study the risk factors and outcome for non-communicable disease. This study consists of two phases: first, the cross sectional study (1999-2001) in which, 15005 individuals, aged over 3 years, and the second (2001-2003, $\mathrm{n}=3551)$ a prospective interventional ongoing phase scheduled to continue for at least 20 years, with regular follow-up data collection at 3-year intervals. Details on the rationale, sampling and data collection of the TLGS have been published previously.

Of a total 9752 participants, aged $\geq 30$ years who enrolled for the TLGS in first or second phase, individuals with a history of $\mathrm{CHD}(\mathrm{N}=522)$ were enrolled in the present study; of these, we excluded 65 (no follow-up information), 8 (missing data on waist circumstance) and 9 (without fasting plasma glucose); the present analysis included 440 TLGS participants with at least one year of follow up (Figure 1). The ethics committee of the research institute for endocrine sciences, Shahid Beheshti University of Medical Sciences, approved the design of the TLGS study, and all participants provided written informed consent.

\subsection{Data Collection}

Baseline information of the TLGS included an interview and completion of a pre-tested self-administered standard and questionnaire. Trained research staff conducted faceto face interviews to collect information on demographics, cigarette smoking, education and medical and drug history, CHD history, physical examination for blood pressure, pulse rate, ECG and anthropometrical measures and laboratory measurements for lipid and glucose profiles; missing information on history of drugs was considered as "no" answer.

All anthropometric measurements were measured using standard protocols; waist circumference (WC) was measured at the level of umbilicus; body mass index was computed by dividing weight (kilogram) to the square of height (meter). In the current study, patients were categorized with respect to their BMI status, normal (BMI $<25$ ), overweight $(25 \leq \mathrm{BMI}<30)$, and obese (BMI $\geq 30)$, based on international cut off points for BMI. A current smoker was defined as a person who smokes cigarettes daily or occasionally and a past smoker, was formerly a daily or occasional smoker who currently does not smoke (14). Vigorous physical activity at least 3 days per week was considered as high physical activity, based on the Lipid Research Clinic (LRC) questionnaire (17). Systolic and diastolic blood pressure (SBP, DBP) were defined, based on the average of the two measurements in a seating position measured on the right arm, using standard mercury. Blood samples collected after 12-14h overnight fasting between 7:00 to 9:00 A.M during physical examinations. Description of biochemical measurement methods for total cholesterol (TC), high density lipoprotein cholesterol (HDL-C), triglycerides (TG) and fasting plasma glucose (FPG) were published elsewhere (14).

For this study, CHD was defined as self-reported positive history of CHD. Self-reported history of CHD was defined as a positive answer at the time of the interview to the question as to whether the patient had ever been given a prior diagnosis of CHD by a physician.

\subsection{Outcome}

In this study, CHD including cases of definite myocardial infarction (MI) (diagnosed by ECG and biomarkers), probable MI (positive ECG findings plus cardiac symptoms or signs or positive ECG findings plus equivocal biomarkers), unstable angina (new cardiac symptoms or changing symptom patterns and positive ECG findings with normal biomarkers), and angiographic proven CHD. Details of outcome data collection have been published elsewhere $(5,14$, 18). 


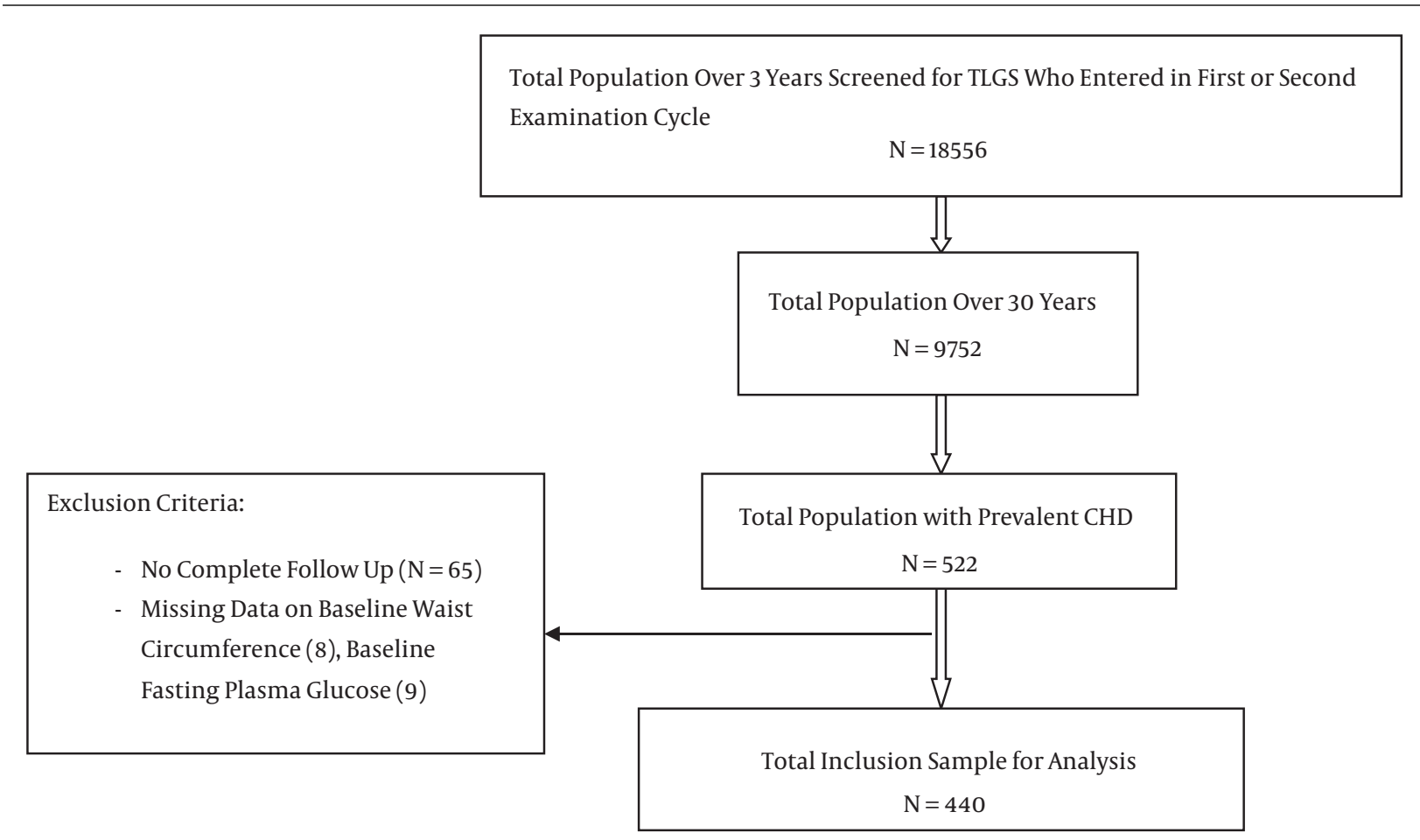

Figure 1. Flow Chart of Study Population

\subsection{Statistical Analysis}

All continuous data with normal distribution are expressed as mean (SD) and skewed parameters as median, interquartile (IQ range), and categorical variables are expressed as percentage. Differences for continuous variables were assessed using the t-test, whereas differences for categorical variables were assessed with the Chi Square Test. The Mann-Whiteny test was used for TG as non-normal variables. For ordinal data, the non-parametric Wilcoxon rank-sum test was used to analyses differences among groups. Participants with CHD history at baseline were followed until the occurrence of a recurrent CHD, the exact date of which was considered as the date of the end point event or death or loss to follow-up, in which case the date of the last patient visit or the date of death due to a nonrecurrent CHD event; were considered as censoring. We calculated incidence rates and adjusted hazard ratios of recurrent CHD events.

The person time for each participant was calculated from the beginning of the study to the date of recurrent CHD event; the date of censoring or the end of the study, whichever came first. Incidence rates of recurrent CHD for each BMI category were obtained by dividing the number of cases by person-years in BMI groups; the Kaplan-Meier method was used to calculate crude cumulative incidence curve for recurrent CHD events. Cox-proportional hazard regression was used to study the effect of BMI groups in the presence of different covariates; assumption of the proportionality was tested based on Schoenfeled residuals. The first model was adjusted for both age and gender and in model 2, we further adjusted was done for waist circumference. In the third model, additional adjustment for current and past smoker, physical activity, education, ACE inhibitor, B-blocker, diabetic, antihypertensive and lipid lowering drug, FPG, triglyceride, high density lipoprotein, total cholesterol, non HDL-C. A forward stepwise approach was considered to keep significant covariates with a pvalue of $<0.2$ for 'enter' and p-value $>0.05$ for 'remove'. Also univariate analysis was carried out to evaluate the interaction effect of gender, past smoker and current smoker interaction effects with BMI categories. No evidence of this was observed. All analysis was conducted using STATA 11 statistical software

\section{Results}

Of a total of 522 individuals, aged $\geq 30$ years, with history of CHD at baseline, 440 (84.29\%) individuals with a mean (SD) age of $59.6(10.8)$ years at baseline were included in the analyses. Over a median (IQ25-75) of 8.44 (3.26-9.94) 
years, 169 cases of recurrent CHD developed and the incidence density of recurrent CHD was calculated as 54.53 per 1000 person-years.

Baseline characteristics of the TLGS study sample population are summarized in Table 1 with respect to their recurrent CHD events; women constitute the majority of the population without recurrent CHD (50.2\% vs. 38.5\%, P-value $=0.02$ ). Generally, the recurrent CHD group had more unfavorable metabolic risk profiles than the nonrecurrent the no recurrence group, but the differences were not statistically significant in simple univariate analysis, except for current and past smoking (P-value $=0.008$ and 0.007, respectively), diabetes lowering drugs (P-value $=0.01)$, aspirin $(\mathrm{P}$-value $=0.01)$ and higher level of FPG $(\mathrm{P}-$ value $=0.046$ ).

The incidence of recurrent CHD was higher in normal BMI (68.71per 1,000 person-year), compared with overweight individuals (47.56 per 1,000 person-year) and obese (54.46 per 1,000 person-year) category (Figure 2 ). Since there was no interaction between gender and the exposure of interest, all analyses was performed, regardless of gender segregation. Results from the Cox proportional hazard models for CHD risk assessment with prior CHD history at time of first examination are shown in Table 2. After adjustment for age, gender and WC, HRs of both the overweight (0.51 95\% CI, 0.32-0.80) and obese (0.52 95\% CI, 0.28-0.99) groups were protective. In model 3 after including other variables, HR of the overweight group remained protective (0.48 95\% CI, 0.30-0.80), whereas that of the obese group was not statistically significant (0.55 95\% CI, 0.28-1.06).

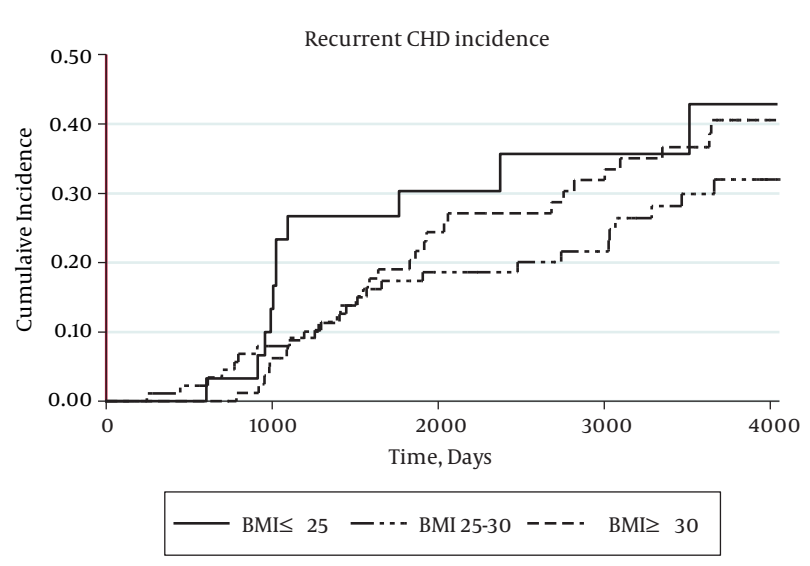

$\overline{\text { Figure 2. Crude Cumulative Incidence of Recurrent CHD Among Different BMI Cat- }}$ egories

\section{Discussion}

In this study of individuals with a history of CHD at baseline and a follow-up at 8.44 median years, we found that the incidence of recurrent CHD was higher in normal BMI $\left(\mathrm{BMI}<25 \mathrm{Kg} / \mathrm{m}^{2}\right)$ than overweight $(25 \leq \mathrm{BMI}<30)$ and obese $\left(\mathrm{BMI} \geq 30 \mathrm{Kg} / \mathrm{m}^{2}\right)$ individuals in adjusted analyses. Moreover, after further adjustment for potential confounders, overweight (but not obese) subjects had a lower risk of recurrent $\mathrm{CAD}$.

These data are consistent with those of previous studies and meta-analyses. Romero-Corral et al. (13) in a metaanalysis of 250,152 patients a mean follow-up of 3.8 years reported a better outcomes for cardiovascular and total mortality in the overweight and mildly obese groups; they included patients with CAD [defined as a history of percutaneous coronary intervention (PCI), coronary artery bypass graft (CABG), or MI] at baseline and reported obesity to be associated with a higher total mortality only in patients with history of CABG. In another meta-analysis involving patients after coronary revascularization procedures (PCI/ CABG), Sharma et al. (18) reported that the risk of total mortality, CVD mortality and MI was highest among patients with low BMI $\left(<25 \mathrm{~kg} / \mathrm{m}^{2}\right)$ at the end of a mean follow-up period of 1.7 years. Moreover, the protective effect of the obesity paradox in patients with acute coronary syndrome (ACS) has been confirmed by Niedziela et al. in recent metaanalysis (19). In their unadjusted analyses performed on data from studies, they confirmed better survival in the overweight, obese and severe obesity group in 16 out of 26 studies, 19 of 26 and 5 of 10 studies, respectively. After adjustment for various co-variables (BMI groups), significant relation was found between lower BMI and worse survival in 15 out of 25 studies. Given the consistency in these data, biological reasons rather than statistical and methodological biases should be responsible for the observed paradox (20). Similar to above mentioned studies and metaanalysis, our population based study showed that patients with past history of CHD who were overweight had lower rates of recurrent CHD events, compared to normal weight ones. Interestingly, when the effect of WC was taken into account, the protective role of general obesity come into effect, indicating that if WC remains constant, the obesity paradox phenomenon can be easily observed, or controversy failing to adjust for the confounder effect of WC may mask protective effect of increasing BMI.

researchers investigating the obesity paradox investigators suggest several possible explanations for better outcomes in overweight or obese patients; first, BMI cannot discriminate between body fat and lean body mass; a high BMI does not necessarily indicate excess body fat and may be attributable to higher lean body mass associ- 
Table 1. Characteristics of TLGS Study Participants With a History of CHD by Their Recurrent Event ${ }^{\mathrm{a}, \mathrm{b}}$

\begin{tabular}{|c|c|c|c|c|}
\hline & Total Study Population ( $\mathrm{N}=\mathbf{4 4 0}$ ) & Without Recurrent CHD Event $(N=271)$ & Recurrent CHD Event $(\mathrm{N}=169)$ & P Value \\
\hline \multicolumn{5}{|l|}{ Gender, No. (\%) } \\
\hline Female & $201(45.7)$ & $136(50.2)$ & $65(38.5)$ & 0.018 \\
\hline Age, $y$ & $59.62(10.83)$ & $59.03(11.84)$ & $60.54(8.92)$ & 0.13 \\
\hline Education & & & & 0.85 \\
\hline$<6, y$ & $286(65.0)$ & $177(65.3)$ & $109(64.5)$ & \\
\hline $6-12, y$ & $129(29.3)$ & $79(29.2)$ & $50(29.6)$ & \\
\hline$\geq 12, y$ & $25(5.7)$ & $15(5.5)$ & $10(5.9)$ & \\
\hline \multicolumn{5}{|l|}{ Family History of CVD } \\
\hline Father & $61(13.9)$ & $36(13.3)$ & $25(14.9)$ & 0.67 \\
\hline Mother & $64(14.5)$ & $34(12.5)$ & $30(17.9)$ & 0.13 \\
\hline Current smokers, No. (\%) & $65(14.8)$ & $30(11.1)$ & $35(20.7)$ & 0.008 \\
\hline Past smoker, No. (\%) & $111(25.2)$ & $56(20.7)$ & $55(32.5)$ & 0.007 \\
\hline BMI, $\mathrm{kg} / \mathrm{m}^{2}$ & $27.82(4.57)$ & $27.87(4.3)$ & $27.74(4.95)$ & 0.78 \\
\hline BMI group, No. (\%) & & & & 0.16 \\
\hline Normal weight & $111(25.2)$ & $61(22.5)$ & $50(29.6)$ & \\
\hline Obesity & $205(46.6)$ & $135(49.8)$ & $70(41.4)$ & \\
\hline Overweight & $124(28.2)$ & $75(27.7)$ & $49(29.0)$ & \\
\hline wC, $\mathbf{c m}$ & $94.06(10.37)$ & $93.67(10.37)$ & $94.7(10.38)$ & 0.33 \\
\hline Abdominal obesity ${ }^{\mathbf{C}}$, No. (\%) & $217(49.3)$ & $130(48.7)$ & $85(50.3)$ & 0.8 \\
\hline $\mathrm{TC}, \mathbf{m g} / \mathbf{d L}$ & $230.28(49.48)$ & $227.97(48.88)$ & $233.99(50.36)$ & 0.21 \\
\hline HDL, mg/dL & $40.85(10.83)$ & $40.81(10.41)$ & $40.91(11.5)$ & 0.92 \\
\hline TG, mg/dL ${ }^{\mathbf{d}}$ & $181.0(119.0)$ & $178.0(117.0)$ & $181.0(122.5)$ & 0.6 \\
\hline Non HDL-C & $189.44(47.63)$ & $187.16(47.42)$ & $193.08(47.89)$ & 0.2 \\
\hline SBP, mmHg & $132.08(22.24)$ & $131.75(21.41)$ & $132.6(23.56)$ & 0.7 \\
\hline DBP, mmHg & $80.1(11.85)$ & $80.73(10.67)$ & $79.09(13.5)$ & 0.16 \\
\hline FPG, $\mathrm{mg} / \mathrm{dL}$ & $116.34(46.09)$ & $112.77(43.76)$ & $122.05(49.21)$ & 0.046 \\
\hline 2h PCG, mg/dL & $145.67(76.12)$ & $141.26(66.84)$ & $153.44(90.04)$ & 0.2 \\
\hline Creatinine & $1.17(0.2)$ & $1.16(0.21)$ & $1.17(0.18)$ & 0.55 \\
\hline \multicolumn{5}{|l|}{ Physical activity } \\
\hline More than 3 times a week & $68(15.5)$ & $43(19.2)$ & $25(17.2)$ & 0.68 \\
\hline \multicolumn{5}{|l|}{ Medication use, No. (\%) } \\
\hline Hypertension drugs & $181(41.1)$ & $108(39.9)$ & $73(43.2)$ & 0.55 \\
\hline Diabetes drugs & $85(19.3)$ & $42(15.5)$ & $43(25.4)$ & 0.01 \\
\hline Lipid lowering drugs & $56(12.7)$ & $32(11.8)$ & $24(14.2)$ & 0.46 \\
\hline Beta blocker drugs & $209(47.5)$ & $132(48.7)$ & $77(45.6)$ & 0.52 \\
\hline ACE inhibitor drugs & $67(15.2)$ & $41(15.1)$ & $26(15.4)$ & 0.94 \\
\hline Aspirin & $236(53.6)$ & $132(48.7)$ & $104(61.5)$ & 0.01 \\
\hline
\end{tabular}

ated with a better prognosis in patients with CVD. Second, higher BMI groups have more recognizable and aggressively treated co-morbidities, like hypertension, hyperlipidemia, and diabetes mellitus allowing for more cardiac medications rather than those with a normal BMI. Third, another possibility is genetic selection bias, in that some overweight and obese persons may have not developed CHD in the first place if weight gain had been prevented, whereas the thin patients who still develop the sme CHD do so for other reasons (e.g., genetic predisposition) that could be associated with a worse prognosis, despite having a more favorable overall CHD risk factor profile.

Several study limitations should be considered in the interpretation of the results. First limitations of this study include a relatively small sample size and low rate of fatal events, which limits reporting of all-cause mortality and 
Table 2. Hazard Ratios (95\% CIs) of BMI Categories for Recurrent CHD Events ${ }^{\mathrm{a}}$

\begin{tabular}{|c|c|c|c|c|c|c|c|c|}
\hline & \multicolumn{2}{|c|}{ Recurrent CHD } & \multirow[t]{2}{*}{ Person/Years } & \multirow{2}{*}{$\begin{array}{c}\text { Incidence (Per 1,000 } \\
\text { person/years) }\end{array}$} & \multirow[t]{2}{*}{ HR, 95\% CI } & \multirow[t]{2}{*}{ PValue } & \multirow[t]{2}{*}{ Harrell's C } & \multirow[t]{2}{*}{ AIC } \\
\hline & Cases, No. & Total, No. & & & & & & \\
\hline Model 1 & & & & & & & 0.56 & 1818.61 \\
\hline $\mathrm{BMI}<25$ & 50 & 111 & 727.68 & 68.71 & Ref & & & \\
\hline BMI 25 - 30 & 70 & 205 & 1471.78 & 47.56 & $0.71(0.5-1.04)$ & 0.077 & & \\
\hline $\mathrm{BMI} \geq 30$ & 49 & 124 & 899.69 & 54.46 & $0.97(0.63-1.5)$ & 0.88 & & \\
\hline Age, y & & & & & $1.01(1.0-1.03)$ & 0.08 & & \\
\hline Gender, Female, ref & & & & & $0.74(0.53-1.04)$ & 0.08 & & \\
\hline Model 2 & & & & & & & 0.59 & 1813.88 \\
\hline $\mathrm{BMI}<25$ & 50 & 111 & 727.68 & 68.71 & Ref & & & \\
\hline BMI 25 - 30 & 70 & 205 & 1471.78 & 47.56 & $0.51(0.32-0.8)$ & 0.003 & & \\
\hline $\mathrm{BMI} \geq 30$ & 49 & 124 & 899.69 & 54.46 & $0.52(0.28-0.99)$ & 0.046 & & \\
\hline Age, y & & & & & $1.0(0.99-1.02)$ & 0.22 & & \\
\hline Gender, Female, ref & & & & & $0.77(0.55-1.08)$ & 0.13 & & \\
\hline $\mathrm{WC}, \mathrm{cm}$ & & & & & $1.03(1.007-1.05)$ & 0.01 & & \\
\hline $\begin{array}{l}\text { Model } 3 \text { (Forward } \\
\text { stepwise model) }\end{array}$ & & & & & & & 0.66 & 1791.33 \\
\hline $\mathrm{BMI}<25$ & 50 & 111 & 727.68 & 68.71 & Ref & & & \\
\hline BMI 25-30 & 70 & 205 & 1471.78 & 47.56 & $0.48(0.3-0.80)$ & 0.004 & & \\
\hline $\mathrm{BMI} \geq 30$ & 49 & 124 & 899.69 & 54.46 & $0.55(0.28--1.06)$ & 0.07 & & \\
\hline WC, $\mathrm{cm}$ & & & - & - & $1.03(1.0-1.05)$ & 0.01 & & \\
\hline Past smoker & & & - & - & $1.76(1.27-3.0)$ & 0.002 & & \\
\hline Current smoker & & & - & - & $1.95(2.24-9.75)$ & $<0.001$ & & \\
\hline Aspirin drug & & & - & - & $1.68(1.20-2.37)$ & 0.003 & & \\
\hline Diabetes drug & & & - & - & $2.11(1.45-3.06)$ & $<0.001$ & & \\
\hline
\end{tabular}

Abbreviations: AIC, akaike information criterion; BMI, body mass index, $\mathrm{kg} / \mathrm{m}^{2}$; DBP, diastolic blood pressure, mmHg; FPG, fasting plasma glucose, mg/dL; HDL-C; highdensity lipoprotein cholesterol, mg/dL; 2h-PCG, 2 h post-challenge plasma glucose, mg/dL; SBP, systolic blood pressure, mmHg; TC, total cholesterol, mg/dL; TG, triglycerides, $\mathrm{mg} / \mathrm{dL}$; WC, waist circumference, $\mathrm{cm}$.

${ }^{a}$ Model 1: BMI group, age, gender; model 2: model 1 + Waist circumference; Model 3: model 2 + (SBP, DBP, TG, TC, HDL, non-HDL, FPG, current and past smoking, physical activity, education, ACE inhibitor, $\beta$-Blocker and aspirin drug, lipid, diabetes, and hypertension drugs).

CHD mortality. Second, we did not perform ECG or angiography to confirm CHD for all individuals. Third, although we adjusted for possible confounders, unmeasured confounding factors associated with BMI may exist. Fourth, we used baseline BMI for analysis and did not measure weight change. Additionally, we were unable to analyze the outcomes for extreme BMI levels due to low number of subjects in this subgroup. We did not measure body composition using bioelectrical impedance which is a better tool for determination of total body fat than BMI

The strength of this study includes prospectively collected data of consecutive unselected patients during a follow-up to evaluate the long term effects of BMI on CHD outcomes. Moreover, we measured WC as a surrogate for central obesity, which is more closely related to adiposityrelated outcomes. We also adjusted for numerous confounding factors were included in the multivariable analysis.

\subsection{Conclusion}

In conclusion, further studies with different ways to identify obesity are needed to fully understand the impacts responsible for the lower cardiovascular events and mortality in overweight/obese patients. Therefore, further studies taking into account the body composition might provide an interesting basis for investigating whether adipose tissue, now recognized as an endocrine organ could facilitate a direct protective effect. 


\section{Acknowledgments}

We express appreciation to the participants of district 13, Tehran, for their enthusiastic support. The authors also wish to acknowledge Mrs Niloofar Shiva for critical editing of the English grammar and syntax of the manuscript.

\section{Footnote}

Authors' Contribution: Conception and design: Samaneh Asgari, Davood Khalili, Farzad Hadaegh, and Farhad Hosseinpanah; drafting the article: Samaneh Asgari, Davood Khalili, Farzad Hadaegh, Farhad Hosseinpanah, Maryam Barzin, and Fereidoun Azizi. All authors contributed to the writing of the paper and read and approved the final manuscript.

\section{References}

1. Guh DP, Zhang W, Bansback N, Amarsi Z, Birmingham CL, Anis AH. The incidence of co-morbidities related to obesity and overweight: a systematic review and meta-analysis. BMC Public Health. 2009;9:88. doi: 10.1186/1471-2458-9-88. [PubMed: 19320986].

2. Jensen MD, Ryan DH, Apovian CM, Ard JD, Comuzzie AG, Donato KA, et al. 2013 AHA/ACC/TOS guideline for the management of overweight and obesity in adults: a report of the American College of Cardiology/American Heart Association Task Force on Practice Guidelines and The Obesity Society. J Am Coll Cardiol. 2014;63(25 Pt B):2985-3023. doi:10.1016/j.jacc.2013.11.004. [PubMed: 24239920].

3. Inoue-Choi M, Lazovich $\mathrm{D}$, Prizment $\mathrm{AE}$, Robien $\mathrm{K}$. Adherence to the World Cancer Research Fund/American Institute for Cancer Research recommendations for cancer prevention is associated with better health-related quality of life among elderly female cancer survivors. J Clin Oncol. 2013;31(14):1758-66. doi: 10.1200/JCO.2012.45.4462. [PubMed: 23569318].

4. D'Agostino RB, Russell MW, Huse DM, Ellison RC, Silbershatz H, Wilson PW, et al. Primary and subsequent coronary risk appraisal: new results from the Framingham study. Am Heart J. 2000;139(2 Pt 1):27281. [PubMed: 10650300].

5. Hadaegh F, Harati H, Ghanbarian A, Azizi F. Prevalence of coronary heart disease among Tehran adults: Tehran Lipid and Glucose Study. East Mediterr Health J. 2009;15(1):157-66. [PubMed:19469439].

6. Khalili D, Sheikholeslami FH, Bakhtiyari M, Azizi F, Momenan AA, Hadaegh F. The incidence of coronary heart disease and the population attributable fraction of its risk factors in Tehran: a 10-year population-based cohort study. PLoS One. 2014;9(8):eee105804. doi: 10.1371/journal.pone.0105804. [PubMed: 25162590].

7. Hadaegh F, Mohebi R, Cheraghi L, Tohidi M, Moghaddam NB, Bozorogmanesh $\mathrm{M}$, et al. Do different metabolic syndrome definitions predict cerebrovascular events and coronary heart disease independent of their components?: 9 years follow-up of the tehran lipid and glucose study. Stroke. 2012;43(6):1669-71. doi: 10.1161/STROKEAHA.112.650812. [PubMed: 22382161].
8. Uretsky S, Messerli FH, Bangalore S, Champion A, Cooper-Dehoff RM, Zhou Q, et al. Obesity paradox in patients with hypertension and coronary artery disease. Am J Med. 2007;120(10):863-70. doi: 10.1016/j.amjmed.2007.05.011. [PubMed: 17904457].

9. Younge JO, Damen NL, van Domburg RT, Pedersen SS. Obesity, health status, and 7-year mortality in percutaneous coronary intervention: in search of an explanation for the obesity paradox. Int $J$ Cardiol. 2013;167(4):1154-8. doi: 10.1016/j.ijcard.2012.03.105. [PubMed: 22475846].

10. Bucholz EM, Rathore SS, Reid KJ, Jones PG, Chan PS, Rich MW, et al. Body mass index and mortality in acute myocardial infarction patients. Am J Med. 2012;125(8):796-803. doi: 10.1016/j.amjmed.2012.01.018. [PubMed: 22483510].

11. Fonarow GC, Srikanthan P, Costanzo MR, Cintron GB, Lopatin M, Adhere Scientific Advisory Committee, et al. An obesity paradox in acute heart failure: analysis of body mass index and inhospital mortality for 108,927 patients in the Acute Decompensated Heart Failure National Registry. Am Heart J. 2007;153(1):74-81. doi: 10.1016/j.ahj.2006.09.007. [PubMed: 17174642].

12. Herment A, Giovannelli JF. An adaptive approach to computing the spectrum and mean frequency of Doppler signals. Ultrason Imaging. 1995;17(1):1-26. [PubMed: 7638930].

13. Romero-Corral A, Montori VM, Somers VK, Korinek J, Thomas RJ, Allison TG, et al. Association of bodyweight with total mortality and with cardiovascular events in coronary artery disease: a systematic review of cohort studies. Lancet. 2006;368(9536):666-78. doi: 10.1016/S01406736(06)69251-9. [PubMed: 16920472].

14. Azizi F, Ghanbarian A, Momenan AA, Hadaegh F, Mirmiran P, Hedayati $M$, et al. Prevention of non-communicable disease in a population in nutrition transition: Tehran Lipid and Glucose Study phase II. Trials. 2009;10:5. doi: 10.1186/1745-6215-10-5. [PubMed: 19166627].

15. Khalili. D, KMosavi-Jarrahi A, Eskandari F, Mousavi-Jarrahi Y, Hadaegh F, Mohagheghi M, et al. Evaluation of cause of deaths' validity using outcome measures from a prospective, population based cohort study in Tehran, Iran. PLoS One. 2012;7(2):e31427. doi: 10.1371/journal.pone.0031427. [PubMed: 22355365].

16. Wilson PW, D’Agostino RS, WBhatt DL, Eagle K, et al. An international model to predict recurrent cardiovascular disease. Am J Med. 2012;125(7):695-703. doi: doi: 10.1016/j.amjmed.2012.01.014.. [PubMed: 22727237].

17. Ainsworth BE, Jacobs DR, Leon AS. Validity and reliability of selfreported physical activity status: the Lipid Research Clinics questionnaire. Medicine and science in sports and exercise. 1993;25(1):92-98.

18. Sharma A, Vallakati A, Einstein AJ, Lavie CJ, et al. Relationship of body mass index with total mortality, cardiovascular mortality, and myocardial infarction after coronary revascularization: evidence from a meta-analysis. Mayo Clin Proc. 2014;89(8):1080-100. doi: 10.1016/j.mayocp.2014.04.020. [PubMed: 25039038].

19. Niedziela J, Hudzik B, Niedziela N, Gasior M, et al. The obesity paradox in acute coronary syndrome: a meta-analysis. Eur J Epidemiol. 2014;29(11):801-12. doi: 10.1007/s10654-014-9961-9. [PubMed: 25354991].

20. Kalantar-Zadeh K, Rhee CM, Amin AN. To legitimize the contentious obesity paradox. Mayo Clin Proc. 2014;89(8):1033-5. doi: 10.1016/j.mayocp.2014.06.015. [PubMed: 25092364]. 